\title{
A Study on Extent of Counseling by Hospital Pharmacists
}

\section{Raj Kumar Thapa ${ }^{1 \dagger}$, Isha Joshi ${ }^{2}$, Prakriti Thapa ${ }^{2}$, Nashreen Bajracharya ${ }^{2}$, Joshika Neupane $^{2}$, Sushma Thapa ${ }^{2}$, Sita Dhakal ${ }^{3}$, Trishna Acharya ${ }^{3,4 \dagger^{*}}$}

\author{
${ }^{1}$ Department of Pharmacy, Patan Hospital, Lalitpur, Nepal \\ ${ }^{2}$ Department of Pharmacy, Kathmandu University, Dhulikhel, Kavre, Nepal \\ ${ }^{3}$ Department of Pharmacy, National Model College for Advance Learning, Tribhuvan University, \\ Kathmandu, Nepal \\ ${ }^{4}$ Nepal Health Research Council, Ramshah Path, Kathmandu, Nepal \\ $\dagger$ Equal Contributors

\section{* Corresponding Author} \\ Trishna Acharya \\ E-mail: shna.tris@gmail.com
}

\begin{abstract}
Pharmacists can improve adherence to drug therapy in order to help patients participate in their own health care decision. The study aimed to observe the problems faced during dispensing and counseling. A cross-sectional study was carried out in two different hospitals of Nepal. ASHP (American Society of Health-System Pharmacy) 1997 and USP (The United States Pharmacopoeia) 1997 guidelines were used to ensure the completeness of the contents of the counseling. The extent of counseling fulfilled by the pharmacists was observed and recorded. Collected data was compiled, managed and analysed using SPSS and MS Excel. Counseling was observed in total 400 cases (200 from each hospital). It was found that complete information from the guidelines was not provided in both the hospitals. Hospital pharmacists of urban region had provided maximum 5 categories of drug information while it was 6 in sub-urban region out of 14 categories given by USP and 16 categories given by ASHP guidelines. Besides this, none of them had received any counseling regarding precaution, side effects/adverse effects, interactions, contraindication, sensitivity and storage of the medications. Further, it was found that polypharmacy, language, lack of qualified personnel in pharmacy, inadequate time, lack of space in the pharmacy were some of the barriers to counseling found in the study. The minimum requirements of the guidelines were met in both the hospitals. However, the safety aspects of drug information were not provided to the patients. Prioritizing the safe and effective use of medicines should be the first consideration of the pharmacists.
\end{abstract}

\section{KEYWORDS}

Counseling, Hospital Pharmacist, Nepal, Pharmacy 


\section{INTRODUCTION}

Pharmacists can improve the adherence to drug therapy by adopting appropriate strategies including patient counseling, education about the medications they receive in order to help patients participate in their own health care decision. They shall provide accurate and comprehensive information about drugs and drug therapy to health professionals, patients and patient's care givers as appropriate and should act as a vital link between patients and prescribers (Adepu \& Nagavi, 2009; American Society of Health-System, 1996; Sittidach, Chayakul, Silpapojakul, Siripaitoon, \& Pattharachayakul, 2004; Varma, McElnay, Hughes, Passmore, \& Varma, 1999).

Patient counseling is considered as an important component of pharmaceutical care services (Lewis, Lasack, Lambert, \& Connor, 1997). Effective leadership and practice management skills are necessary for delivery of pharmacy services in a manner consistent with a hospital and patient's need (American Society of Health-System, 1995). It is essential to improve the use of medications, enhancements in patients' satisfaction, patient compliance, decrease in treatment time, reduction in adverse effects, decrease in total health care costs and ensure optimal therapeutic outcomes (Ali, Laurin, Lariviere, Tremblay, \& Cloutier, 2002; Gerber, Liu, \& McCombs, 1998; Morrison \& Wertheimer, 2001; Schnipper et al., 2006; Thomson, Cunningham, \& Hunt, 2001).

It's seen that pharmacy practice is in a rudimentary stage in most of the developing countries (Adepu \& Nagavi, 2009). People without academic qualification are allowed to run pharmacies and dispense medicines in Nepal(Mühlich, Scherrer, \& Daschner, 2003). Selfmedication and non-doctor prescription are the common drug use problems in Nepal. Similarly, due to the busy pharmacy and lack of privacy, patients are deprived of receiving proper counseling(Shankar, Partha, \& Shenoy, 2002).

Communication errors are considered as the most common cause of medical errors which can be prevented by well-designed policies (Murphy \& Dunn). Counseling should be done in a structured way. Several guidelines like ASHP (American Society of Health-System Pharmacy), USP (The United States Pharmacopoeia) and OBRA (Omnibus budget reconciliation) etc. have been published regarding counseling the patients during dispensing of medicines. According to USP 1997, pharmacists give the customer sufficient but customized information(Pharmacists, 1997).

Locality of a hospital plays a significant role in counseling the patients. Having an urban or sub-urban location is an important environmental factor for hospitals. The hospitals in suburban regions have less competition and are less expensive than urban regions. Further, pharmacies located in sub-urban regions have less flow of patients comparing to urban regions and there is sufficient time to communicate with the patients. Similarly, the patients visiting hospitals in suburban locations are more relaxed. This is opposite in case of urban regions where there is lack of sufficient time to communicate properly.

The study helped to identify the most frequently counseled contents and most ignored contents of counseling taking reference of two international guidelines. Further, it helped to find 
out the problems faced during dispensing and counseling and to identify the reasons behind poor counseling.

\section{METHODOLOGY}

A cross-sectional study was carried out in two different hospitals of Nepal, one in the sub-urban region (Dhulikhel hospital, Kavre) and other in the urban region (Patan hospital, Kathmandu valley).Ethical approval was granted from both the hospitals to conduct the study. Sample size of total 400 (200 patients from each hospital) was taken.

ASHP 1997 and USP 1997 guidelines were used as the theoretical framework for this study. These guidelines are used to ensure the completeness of the contents of counseling in both the hospitals. The extent of counseling fulfilled by the pharmacists while dispensing was observed in the pharmacies and recording was done on the basis of presence or absence of the information in both the hospitals, one being located in the city area with major population whereas another, in the sub-urban region hospital, located far from Kathmandu valley. Comparison and analysis of counseling pattern were done accordingly in both the hospitals to see any differences in counselingbehavior in both regions.

Standard questionnaire was used as a tool for collection of information. The questionnaire consisted of 3 sections. The first section consisted of ASHP 1997 guidelines contents for pharmacists on counseling patients on prescribed medicines. The contents of the guideline were name and description, therapeutic class, indications, expected benefits, route of administration, dose and dosage form, directions for use, special directions, duration of therapy, adoption into daily routine, precautions, side effects/adverse effects, interactions, contraindications, selfmonitoring techniques and storage. Counseling was observed carefully and then recorded was carefully according to the fulfillment of the contents of counseling according to the guideline.

The second section consisted of USP 1997 guidelines for pharmacists on counseling patients on prescribed medicines. In a similar way, we recorded a tick mark and cross mark based on presence or absence of the contents on observation.

The third section consisted of general information regarding prescription and patient. It included whether prescription is new or repeated, has narrow therapeutic index drugs or not and has complex dosage forms or not.

Collected data was compiled, managed and analysed using Student version of Statistical Package for Social Sciences (SPSS) software in 21.0 versions and MS Excel.

\section{RESULTS}

Counseling was observed in total 400 cases; 200 from each hospital. Among 400 cases, there were total 40 children, 248 adults and 76 elders (Table 1). Out of total patients, multiple medicines were dispensed to maximum $(\mathrm{n}=290,72.5 \%)$ patients including $132(66 \%)$ in urban and $158(79 \%)$

patients in sub-urban region whereas single medicine was dispensed to $110(27.5 \%)$ patients, 68 $(34 \%)$ in urban and $42(21 \%)$ patients in sub-urban region. 
It was found that complete information from the guidelines was not provided in both the hospitals. However, the minimum requirement of guidelines was met. Hospital pharmacists of urban region had provided maximum 5 categories of drugs information while in sub-urban region, maximum 6 categories of drug information were provided out of 14 categories given by USP and 16 categories given by ASHP guidelines.

In addition, information such as route of administration, dose and dosage form, direction for use and duration of therapy were given to $100 \%$ patients in both hospitals. Further, 120 (60.5\%) patients were counseled about name and description of medicines in written form in sub-urban region. In contrast, it was not provided at all in urban region hospital. Special directions were provided to 38 (19\%) patients in sub-urban region and $39(19.5 \%)$ patients in urban region. Additional information from USP guidelines like indication, other treatment option, adoption into daily routine, precaution, side effects/adverse effects, interactions, contraindication, storage was not explained at all in both the hospitals. Similarly, according to the ASHP guidelines, additional information like indications, therapeutics class, expected benefits, adopting into daily routine, selfmonitoring techniques, precaution, side/adverse effects interaction, contraindication, storage were not explained at all in both hospitals. There is no significant difference $(p>0.05)$ in counseling by hospital pharmacists in two hospitals and is independent of the age of the patients too. The differences and similarities of extent of counseling followed as per USP guidelines and ASHP guidelines by both hospitals are presented in the figures (Figure 1 and 2).

It was found that, among 200 patients visiting pharmacy in urban region, 100\% patients could read the label whereas, insub-urban region, only 165 (82.5\%) patients could read the label. Similarly, among total 400 patients, 380 (95\%) patients agreed that they had understood the information clearly while $20(5 \%)$ patients were found confused. Among those 20patients, 12 $(60 \%)$ reported that poly-pharmacy is the barrier of understanding while only $8(5 \%)$ patients reported language as the barrier (their ethnicity being Newar). Besides this, inadequate time and lack of space in the pharmacy were some other barriers to counseling.

Moreover, out of total patients, 6 (1.5\%) patients (2 from urban region and 4from suburban region) had received narrow therapeutic index medicines and none of them had received any counseling regarding sensitivity of the medications.

\section{DISCUSSION}

The extent of counseling was observed in two different hospitals from sub-urban and urban region. Although all the information from both the guidelines was not completely provided in both hospitals, the minimum requirement of the guidelines was met. Another similar study was conducted to find the usefulness of the USP Medication Counseling behavior guidelines and according to which, more than half of the respondents were unaware of the guidelines in spite of promoting the guidelines continuously. Also, the guidelines were considered more useful in learning the principles of patient counseling than learning self-evaluation of performance(Puumalainen, Kansanaho, Varunki, Ahonen, \& Airaksinen, 2005). 
The need for counseling regarding drug therapy has been an important part of pharmacy practice and pharmaceutical care. It is the responsibility of the pharmacists to counsel the patients before dispensing the medicines. Health care professionals underestimate patients' desire for information and their ability to understand medical findings (Shapiro \& Morris, 1978). Additionally, a study reported that most of the community pharmacists believed that counseling was necessary as their own duty but less than $50 \%$ only knew that it was necessary to increase patient compliance (Poudel, Khanal, Alam, \& Palaian, 2009).

For each patient, the pharmacists shall determine the amount of counseling that is reasonable and necessary under the circumstance to promote safe and effective administration of the medication and to facilitate an appropriate therapeutic outcome for the patient from the prescription. It is necessary to allocate sufficient time for patient counseling but sometimes large number of patients per pharmacists or patient eagerness for the rapid discharge from the hospital may contribute as the barrier for effective counseling (Albekairy, 2014). In our study, too many pharmacists expressed that counseling became very difficult during peak business hours due to excessive workload and lack of privacy. Analysis of poor patient counseling showed that inadequate time, lack of space in the pharmacy, poly-pharmacy and language were some barriers to counseling. According to a study conducted in Nepal, lack of time was found as the major barrier. The number of pharmacists should be increased in order to overcome the barriers in Nepal (Poudel et al., 2009). Another similar study conducted in Nepal found language as the major barrier for better counseling (Mishra et al., 2005). The barriers to counseling found in this study are also similar to various other studies (Adepu \& Nagavi, 2009; Alomar, Qandil, Al-Hilwani, Malkat, \& Caroline; Mishra et al., 2005; Poudel et al., 2009; Raisch, 1993; Sokar-Todd \& Einarson, 2003).

Most of the patients require good and private counseling but private counseling rooms are not available in pharmacies of Nepal. Due to busy pharmacy and lack of privacy in hospital pharmacy, patients are deprived of receiving the proper counseling though standard criteria to be fulfilled during counseling have been published by different guidelines(Poudel et al., 2009; Wachter, Joshi, \& Rimal, 1999).

Effective communication by pharmacists is essential to improve the use of medications by patients and ensure the optimal therapeutic outcomes. Total health-care costs can be decreased by pharmacists consultations(Gerber et al., 1998). A study has reported that pharmacist counselingreduces preventable adverse effects in patients from 11 to 1\% (Schnipper et al., 2006). However, in this study, none of the patients had received any counseling regarding multiple drug therapies, sensitivity of their medication, indication, expected benefit, precaution, side effects, contraindication and storage condition. Different studies have also concluded that when patients understand information that is communicated by the health-care providers, there are significant enhancements of patient satisfaction, compliance and outcomes and decrease of treatment time (Thomson et al., 2001).

\section{CONCLUSIONS}


The minimum requirement of the guidelines was met in both the hospitals. However, the safety aspects of drug information were not provided to the patients. It was found that hospital pharmacists provided similar kinds of information despite their location. Prioritizing the safe and effective use of medicines should be the first consideration of the pharmacists. Further, updating pharmacy policies will empower pharmacists to offer and allow time for patient counseling and education.

\section{RECOMMENDATIONS}

More efforts to educate the pharmacists about their role in dispensing, along with increased enforcement of existing regulations must be followed in Nepal. Therefore, there is greater need to advocate and conduct research concerning long term health promotion and public education through the pharmacy professionals. Communication efforts of the pharmacists can be assisted by educating patients about the role of the pharmacists as the source of information on medicine by promoting pharmacist intervention and creating college curriculum that include communication skills and strategies as an important component.

\section{LIMITATION OF THE STUDY}

The total number of pharmacists studied was low and hence, our findings may not reflect the perception of the entire pharmacists in Nepal. Further, the existence of the researchers might affect the counselingbehavior of the pharmacists.

\section{REFERENCES}

Adepu, R., \& Nagavi, B. G. (2009). Attitudes and behaviors of practicing community pharmacists towards patient counselling. Indian journal of pharmaceutical sciences, 71(3), 285.

Albekairy, A. M. (2014). Pharmacists' Perceived Barriers to Patient Counseling. Journal of Applied Pharmaceutical Science, 4(1), 70.

Ali, F., Laurin, M., Lariviere, C., Tremblay, D., \& Cloutier, D. (2002). The effect of pharmacist intervention and patient education on lipid-lowering medication compliance and plasma cholesterol levels. The Canadian journal of clinical pharmacology= Journal canadien de pharmacologie clinique, 10(3), 101-106.

Alomar, M. J., Qandil, S., Al-Hilwani, H. M. A., Malkat, D. M., \& Caroline, C. Evaluation of the community pharmacistâ€ $\mathrm{TM}_{\mathrm{S}}$ behavior towards a prescription of antidiabetic and antiasthma drugs. Pharmacy practice, 9(1), 37.

American Society of Health-System, P. (1995). ASHP guidelines: minimum standard for pharmacies in hospitals. Am J Health-Syst Pharm, 52(23), 2711-2717.

American Society of Health-System, P. (1996). ASHP guidelines on the provision of medication information by pharmacists. Am J Health-Syst Pharm, 53(15), 1843-1845.

Gerber, R. A., Liu, G., \& McCombs, J. S. (1998). Impact of pharmacist consultations provided to patients with diabetes on healthcare costs in a health maintenance organization. The American journal of managed care, 4(7), 991-1000. 
Lewis, R. K., Lasack, N. L., Lambert, B. L., \& Connor, S. E. (1997). Patient counseling--a focus on maintenance therapy. American journal of health-system pharmacy, 54(18), 2084-2098.

Mishra, P., Subish, P., Upadhyay, D., Bista, D., Alam, K., \& Bhandari, R. (2005). Medication counselingcenter in a teaching hospital. Journal of Nepal Medical Association, 44(160).

Morrison, A., \& Wertheimer, A. I. (2001). Evaluation of studies investigating the effectiveness of pharmacists' clinical services. AMERICAN JOURNAL OF HEALTH SYSTEM PHARMACY, 58(7), 569-576.

Mühlich, M., Scherrer, M., \& Daschner, F. (2003). Comparison of infectious waste management in European hospitals. Journal of Hospital Infection, 55(4), 260-268.

Murphy, J. G., \& Dunn, W. F. Medical errors and poor communication. Chest Journal, 138(6), 1292-1293.

Pharmacists, A. S. o. H.-S. (1997). ASHP guidelines on pharmacist-conducted patient education and counseling. Am J Health Syst Pharm, 54(4), 431-434.

Poudel, A., Khanal, S., Alam, K., \& Palaian, S. (2009). Perception of Nepalese community pharmacists towards patient counseling and continuing pharmacy education program: a multicentric study. J Clin Diag Res, 3(2), 1408-1413.

Puumalainen, I., Kansanaho, H., Varunki, M., Ahonen, R., \& Airaksinen, M. (2005). Usefulness of the USP medication counselling behavior guidelines. Pharmacy World and Science, 27(6), 465-468.

Raisch, D. W. (1993). Barriers to providing cognitive services. American pharmacy, 33(12), 5458.

Schnipper, J. L., Kirwin, J. L., Cotugno, M. C., Wahlstrom, S. A., Brown, B. A., Tarvin, E., . . . McKean, S. C. (2006). Role of pharmacist counseling in preventing adverse drug events after hospitalization. Archives of internal medicine, 166(5), 565-571.

Shankar, P., Partha, P., \& Shenoy, N. (2002). Self-medication and non-doctor prescription practices in Pokhara valley, Western Nepal: a questionnaire-based study. BMC family practice, 3(1), 1.

Shapiro, A. K., \& Morris, L. A. (1978). The placebo effect in medical and psychological therapies. Handbook of psychotherapy and behavior change, 2, 369-409.

Sittidach, M., Chayakul, P., Silpapojakul, K., Siripaitoon, P., \& Pattharachayakul, S. (2004). Impact of pharmacist counseling on adherence to antirtroviral therapy. Paper presented at the Int Conf AIDS.

Sokar-Todd, H. B., \& Einarson, T. R. (2003). Community pharmacy practice research: a systematic review of the past 32 years. CPJ, Canadian pharmaceutical journal, 136(8), 2638.

Thomson, A. M., Cunningham, S. J., \& Hunt, N. P. (2001). A comparison of information retention at an initial orthodontic consultation. The European Journal of Orthodontics, 23(2), 169178.

Varma, S., McElnay, J. C., Hughes, C. M., Passmore, A. P., \& Varma, M. (1999). Pharmaceutical care of patients with congestive heart failure: interventions and outcomes. 
Pharmacotherapy: The Journal of Human Pharmacology and Drug Therapy, 19(7), 860869.

Wachter, D. A., Joshi, M. P., \& Rimal, B. (1999). Antibiotic dispensing by drug retailers in Kathmandu, Nepal. Tropical Medicine \& International Health, 4(11), 782-788. 
ISSN: 2362-1303 (Paper) | eISSN: 2362-1311(Online)

JOURNAL OF ADVANCED ACADEMIC RESEARCH (JAAR)

December 2017

Tables and Figures

Table 1: Age distribution of Patients

\begin{tabular}{llll}
\hline Age category & Urban region & Sub-urban region & Total cases \\
\hline Child (<10 years) & $17(8.5 \%)$ & $23(11.5 \%)$ & $40(10 \%)$ \\
Adult (11 to 59 years) & $162(81 \%)$ & $122(61 \%)$ & $284(71 \%)$ \\
Elder (>60 years) & $21(10.5 \%)$ & $55(27.5 \%)$ & $76(18 \%)$ \\
\hline Total & $\mathbf{2 0 0}$ & $\mathbf{2 0 0}$ & $\mathbf{4 0 0}$ \\
\hline
\end{tabular}




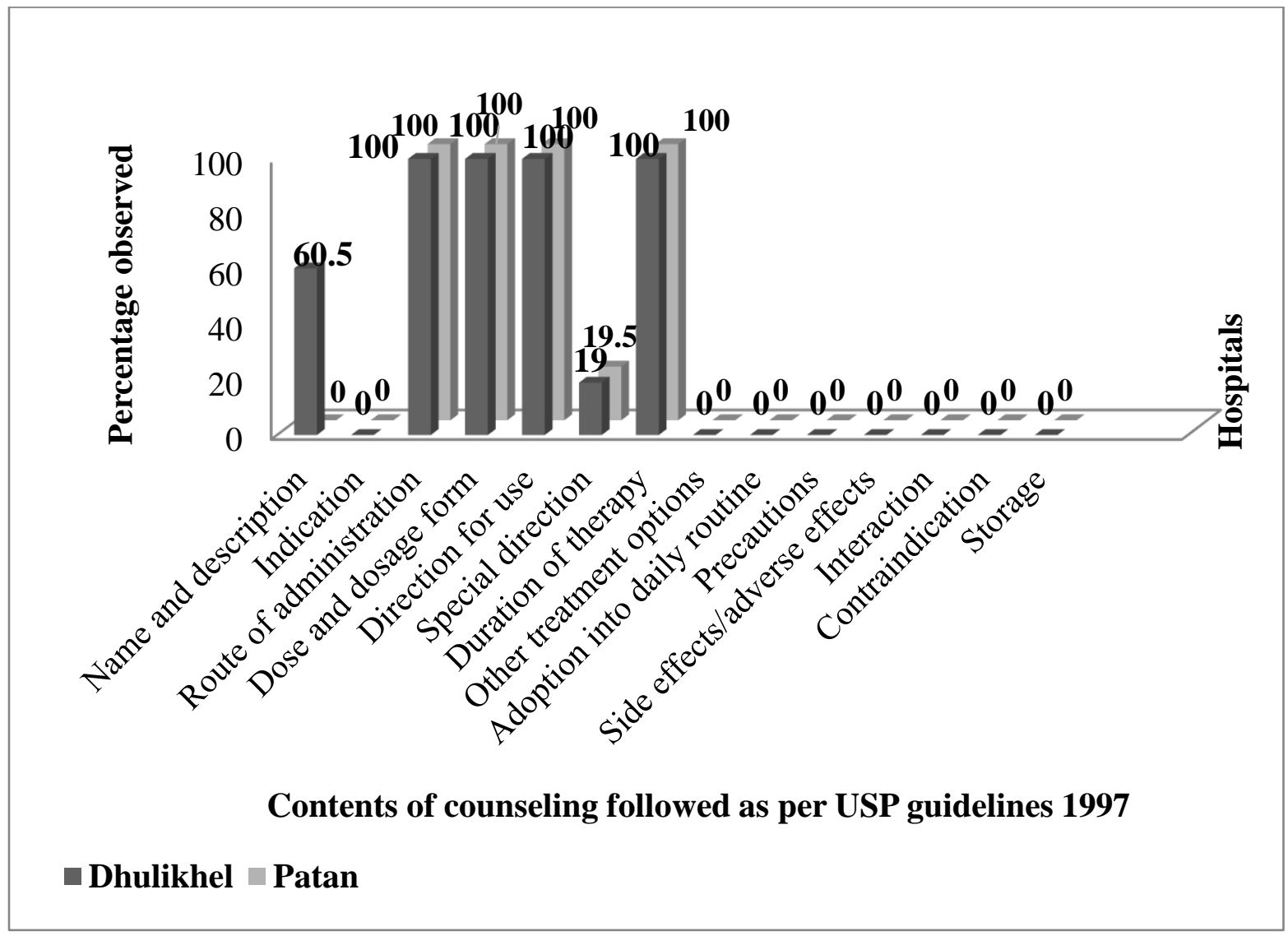

Figure 1: Bar diagram showing the extent of counseling followed as per USP guideline, 1997 


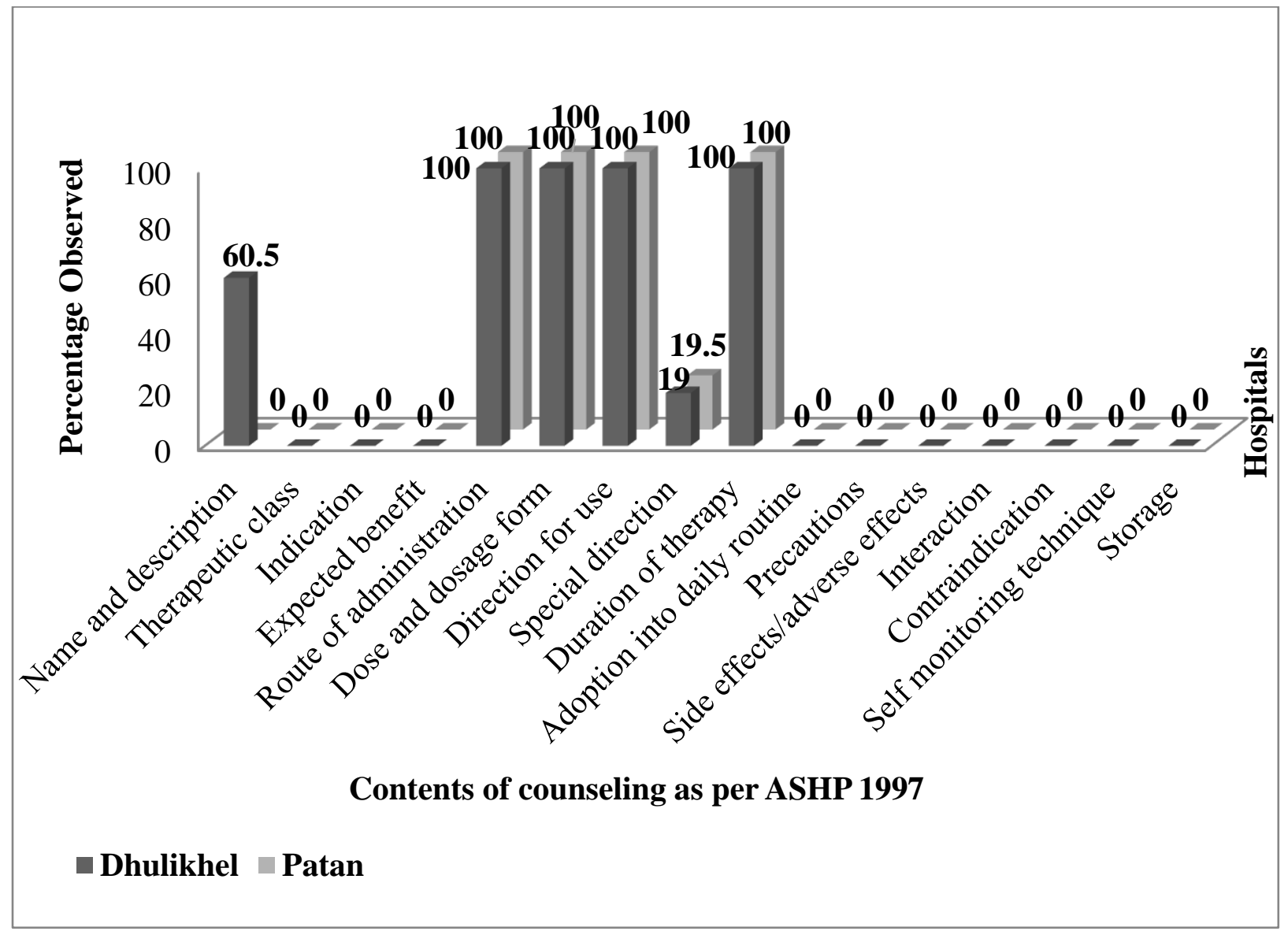

Figure 2: Bar diagram showing the extent of counseling followed as per ASHP guideline, 1997 